Ion trajectories in atom probe field ion microscopy and gas field ion sources

This article has been downloaded from IOPscience. Please scroll down to see the full text article.

1999 J. Phys. D: Appl. Phys. 322261

(http://iopscience.iop.org/0022-3727/32/17/317)

View the table of contents for this issue, or go to the journal homepage for more

Download details:

IP Address: 200.130.19.138

The article was downloaded on $06 / 08 / 2013$ at $18: 47$

Please note that terms and conditions apply. 


\title{
Ion trajectories in atom probe field ion microscopy and gas field ion sources
}

\author{
Caio M C de Castilho \\ Department of Chemistry, University of Cambridge, Lensfield Road, Cambridge CB3 1EW, \\ UK and Instituto de Física, Universidade Federal da Bahia, \\ Campus Universitário da Federação, 40210 - 340 Salvador, Bahia, Brazil \\ E-mail: cmcc@cam.ac.uk and caio@ufba.br
}

Received 8 April 1999, in final form 23 June 1999

\begin{abstract}
Trajectories of positive ions produced in a region close to a structured surface, modelled by spherical or spheroidal protrusions and kept at a positive electric potential with respect to a distant screen or detector are calculated. The results are discussed in comparison with similar practical situations produced by field ionization and field evaporation or desorption, such as those occurring in gas field ion sources, field ion microscopy and field desorption spectroscopy.
\end{abstract}

\section{Introduction}

Determination of the ion trajectories is an important aspect when interpreting results from experiments with the atomprobe field ion microscopy (AP-FIM) and gas field ion sources (GFIS). When dealing with the AP-FIM, the importance of the trajectory determination results from the small displacements observed from field ion microscope (FIM) images and field desorption microscope (FDM) images, which, consequently, affects the aligning process in the AP aperture (Miller et al 1996). Anomalies in ion trajectories, as we compare those produced by field ionization in the FIM or by field desorption or evaporation, were attributed to electric field effects (Brenner and McKinney 1970, Panitz 1978). However, the specific case of field desorption (or evaporation) was also interpreted as a result of the ion acquiring kinetic energy for a movement in specific directions along the surface structure, prior to desorption/evaporation (Waugh et al 1976). When a small protrusion (supertip) is grown on top of a conventional GFIS emitter, the usual half-width angle of the ionic divergent beam is reduced by a factor of ten. This has been interpreted as a result of a 'self-focusing' process caused by the electric field (Jousten et al 1988), which gives then the possibility of a GFIS being 'capable of delivering even 10-100 times higher beam intensities to the image spot', as we compare with the ones produced by a previous technology (Kalbitzer and Knoblauch 1998).

Most of the difficulties in determining the ion trajectories arise from two factors.

(i) The necessity of an adequate model for the electric field in the close vicinity of the sample (AP-FIM) or source (GFIS), where local variations along atomic scale dimensions and in time (when a pulse in the electric potential is applied) affect the subsequent trajectory. (ii) The overall shape of the shank, which in general, does not coincide with models that permit an analytical expression for the field, for example, hyperboloidal or paraboloidal metallic samples. These problems have been discussed by several authors (Miller et al 1996, Smith and Walls 1978, Borisov et al 1968, Gillot and Sugden 1973).

In this paper we present trajectory calculations for ions, originating from a point at the surface of a metal tip and from a point a few angstroms away of it. This would correspond then, respectively, to ions in the operating conditions of a FDM and of either a FIM or of a field ion source. The difference in the arrival positions for the ions, at what would be, a screen or a detector, is calculated and comparisons for simple cases, intended to represent practical situations, are also made.

The remaining part of this paper is as follows. In section 2 we present the mathematical treatment that was used for the electric field and for the numerical determination of the ion trajectories, in section 3 the results are presented and discussed and, finally, in section 4 , some conclusions are drawn.

\section{The mathematical treatment}

An analytical general treatment for ion trajectories at conditions of the FIM and similar apparatus was carried out by Smith and Walls (1978) and a review of the general problem of field intensity and trajectories is given in the book by Miller et al (1996). They considered an electric field in the region between the tip and the screen as being symmetric, with respect to the same symmetry axis of the tip (hyperboloidal or paraboloidal) itself-the $z$-axis in the following. Within this assumption, it is then possible to write 


\section{M C de Castilho}

the electric potential in this region as

$$
V(x, z)=V_{0} \Phi(x, z)
$$

The radial vector to the origin, $r$, and the relationship with the other polar coordinate, $\theta$, is given by

$$
\begin{aligned}
& x=r \sin \theta \\
& z=r \cos \theta
\end{aligned}
$$

An ion of charge ne, with $e$ as the modulus of the electron's charge, is then accelerated by such a potential with

$$
\frac{\mathrm{d} v}{\mathrm{~d} t}=\frac{n e}{m}[-\nabla V(x, z)]
$$

Assuming that the ion is initially at rest at a point where the electric potential is arbitrarily taken as zero leads, with energy conservation, to

$$
|v|^{2}=\frac{2 n e V_{0}}{m}[1-\Phi(x, z)]
$$

Standard determination (Miller et al 1996) of the movement equation leads to

$$
\left|\frac{\mathrm{d} z}{\mathrm{~d} t}\right|^{2}=\frac{2 n e V_{0}}{m}[1-\Phi(x, z)]\left(1+\left|\frac{\mathrm{d} x}{\mathrm{~d} z}\right|^{2}\right)^{-1}
$$

and

$$
\begin{aligned}
& 2[\Phi(x, z)-1] \frac{\mathrm{d}^{2} x}{\mathrm{~d} z^{2}}=\left[\left(\frac{\partial \Phi(x, z)}{\partial x}\right)-\frac{\partial \Phi(x, z)}{\partial z} \frac{\mathrm{d} x}{\mathrm{~d} z}\right] \\
& \times\left(1+\left|\frac{\mathrm{d} x}{\mathrm{~d} z}\right|^{2}\right) .
\end{aligned}
$$

From these equations it is then possible to obtain the ion trajectories by numerical integration.

It is important to emphasize here the importance of these results, if we are interested in comparing the results of FIM and FDM. As can be seen from (6), the ion trajectories are independent of the applied voltage and also are independent of the mass and charge of the ion itself. They are then dependent on the field distribution and on the shape and dimensions characteristics of the sample and of the whole apparatus.

For solving (5) and/or (6), it is necessary to define a specific geometry for the sample and choose the specific shape and position for the screen or detector position.

\subsection{The models for the electric field}

For the ion trajectories we are going to consider in this paper, we have adopted two models for the tip/sample: (a) a hyperboloidal tip with an hemisphere superimposed on the very end of it and (b) a prolate spheroid hemisphere superimposed on a planar surface. Some limiting cases are also discussed and schematic diagrams for the two models are shown in figures 1(a) and (b) respectively.

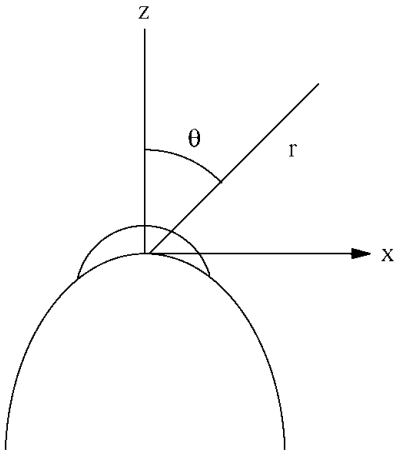

(a)

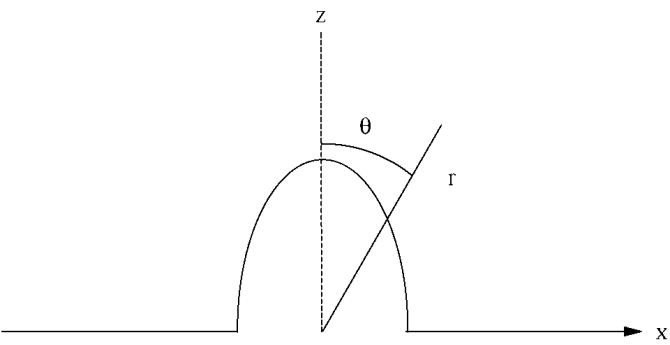

(b)

Figure 1. Schematic drawing for the protrusions models: (a) a hemisphere of radius $R_{s}$ on an otherwise smooth hyperboloid of radius $R_{t}$ and (b) a prolate spheroid of smaller radius $R_{s}$ on a planar surface. In the last case, (b), the ratio between the larger and smaller axis of the spheroid is denoted by $\alpha$.

2.1.1. Model (a) - the hyperboloidal tip with a hemisphere. Let us consider the set-up for a FIM or, alternatively, for a FDM sample, consisting of a hyperboloidal tip with a hemisphere (a half-sphere) superimposed on its apex. The radius of curvature of the hyperboloid, at its apex (without the hemisphere), is denoted below by $R_{t}$ and the radius of the hemisphere by $R_{s}$. Considering the symmetry axis of the tip as being the $z$-axis, with the positive direction outwards from the tip and denoting by $r$ and $\theta$ the usual polar coordinates ( $\theta$ is the angle between $r$ and $z$ - (see figure 1(a)), the electric potential in the region between the tip and the screen can be expressed (de Castilho and Kingham 1986) as

$$
\begin{aligned}
V= & -F_{0}\left[\frac{R_{t}}{2} \ln \left(\frac{\left.R_{t}+2 r \cos \theta\right)}{R_{t}+2 R_{s} \cos \theta}\right)\right. \\
& \left.-R_{s}^{3} \cos \theta\left(\frac{1}{r^{2}}-\frac{1}{R_{s}^{2}}\right)\right]
\end{aligned}
$$

where $F_{0}$ is the electric field at the apex of the hyperboloid without the hemisphere and the tip surface is taken as the loci of points with zero potential.

The $r$ and $\theta$ components of the electric field are then given by

$$
\begin{aligned}
F_{r}= & F_{0}\left[\frac{R_{t} \cos \theta}{R_{t}+2 r \cos \theta}+\frac{2 R_{s}^{3} \cos \theta}{r^{3}}\right] \\
F_{\theta}= & F_{0} \sin \theta\left\{\frac{R_{t} R_{s}}{r\left(R_{t}+2 R_{s} \cos \theta\right)}-\frac{R_{t}}{R_{t}+2 r \cos \theta}\right. \\
& \left.+\frac{R_{s}^{3}}{r^{3}}-\frac{R_{s}}{r}\right\} .
\end{aligned}
$$


2.1.2. Model (b) — the prolate spheroid hemisphere on a planar surface. Considerations of protruding forms on a smooth surface have been adopted by several authors when dealing with theoretical treatments of problems in field emission and field ionization (Rose 1956, Bono and Good 1983, Homeier and Kingham 1983).

To obtain an expression for the electric potential and field components in the region between a plane with a superimposed prolate spheroid, at zero potential and a far away plane with a negative potential (see figure 1(b)), we can use the prolate spheroidal coordinates (Morse and Feshbach 1953, Schelkunoff 1961). Their relation with Cartesian coordinates can be expressed (Homeier and Kingham 1983) as

$$
\begin{gathered}
x=c\left[\left(\xi^{2}-1\right)\left(1-\eta^{2}\right)\right]^{1 / 2} \cos \theta \\
y=c\left[\left(\xi^{2}-1\right)\left(1-\eta^{2}\right)\right]^{1 / 2} \sin \theta \\
z=c \xi \eta .
\end{gathered}
$$

Alternatively, using the most common notation (Morse and Feshbach 1953), this is equivalent to

$$
\begin{gathered}
x=c \sinh \mu \sin v \cos \theta \\
y=c \sinh \mu \sin v \sin \theta \\
z=c \cosh \mu \cos v .
\end{gathered}
$$

The electric potential can be expressed (Homeier and Kingham 1983) as

$$
V=c F_{0} \eta\left(\left|\xi_{0} \frac{0.5 \xi \ln [(\xi+1)(\xi-1)]-1}{0.5 \xi_{0} \ln \left[\left(\xi_{0}+1\right) /\left(\xi_{0}-1\right)\right]-1}-\xi\right|\right)
$$

where

$$
\xi_{0}=\frac{\alpha}{\left(\alpha^{2}-1\right)^{1 / 2}}
$$

and $\alpha$ is the ratio between the greater (longitudinal) and smaller (transversal) dimensions of the spheroid.

The electric field components, $F_{r}$ and $F \theta$, are then calculated from the partial derivatives, i.e. from

$$
\begin{aligned}
& \frac{\partial V}{\partial r}=\left(\frac{\partial V}{\partial \xi} \frac{\partial \xi}{\partial x}+\frac{\partial V}{\partial \eta} \frac{\partial \eta}{\partial x}\right) \frac{\partial x}{\partial r}+\left(\frac{\partial V}{\partial \xi} \frac{\partial \xi}{\partial z}+\frac{\partial V}{\partial \eta} \frac{\partial \eta}{\partial z}\right)\left(\begin{array}{l}
\left.\frac{\partial z}{\partial r}\right) \\
\frac{\partial V}{\partial \theta}
\end{array}=\left(\frac{\partial V}{\partial \xi} \frac{\partial \xi}{\partial x}+\frac{\partial V}{\partial \eta} \frac{\partial \eta}{\partial x}\right) \frac{\partial x}{\partial \theta}+\left(\frac{\partial V}{\partial \xi} \frac{\partial \xi}{\partial z}+\frac{\partial V}{\partial \eta} \frac{\partial \eta}{\partial z}\right) \frac{\partial z}{\partial \theta}\right.
\end{aligned}
$$

\subsection{The trajectories determination}

With the expression for the field components for each model as described above, we numerically calculate the equation of motion from a starting position, where the particle is assumed to be at rest. The time increment used in the numerical calculation is progressively reduced up to a point where a further reduction affects the result in a negligible way. Two possibilities were considered for the starting position:

(i) the particle is at the tip surface (in fact the 'electrical surface'), at a point with angular coordinate $\theta$; and (ii) the particle is at the critical distance (Inghram and Gomer 1954) above the position at the surface and along the same radial direction, i.e. with the same angular position $\theta$ of the previous case. In order to calculate the $r$ coordinate corresponding to the critical distance, we have used the difference between the potential energy at the surface and at a critical distance, which is approximately given by (Homeier and Kingham 1983)

$$
V_{\text {surf }}-V_{\text {crit }}=I-\Phi
$$

where $I$ is the ionization potential of the imaging gas atom and $\Phi$ is the sample's work function.

\section{Results and discussion}

In all the calculations we have performed, the following values were used. For the ionization potential of the gas atoms we have considered $24.6 \mathrm{eV}$ (helium) while $4.47 \mathrm{eV}$ was the value for the work function (tungsten). The intensity for the electric field $F_{0}$ (see previous section) was taken as being $4.0 \mathrm{~V}^{-1}\left(40 \mathrm{~V} \mathrm{~nm}^{-1}\right)$. The mass of the gas atom was $4.0 \mathrm{u}$ (unified atomic mass of helium) and the ion's charge was +1.0 e (elementary charge). The distance between sample and screen (or detector) was taken, in all the calculations, as $12 \mathrm{~cm}$.

Among the other tests performed in order to verify the consistency of the computational programs used, we did the calculations for a situation where the two models should give the same results. This would correspond, considering the value of $R_{t}$, as significantly bigger that the value of $R_{s}$ in the model of a hemisphere superimposed on an hyperboloidal apex. A ratio $R_{t} / R_{s}$ equal to $10^{7}$ was used, which would correspond, in practical terms, to a hemisphere superimposed on a planar surface. On the other hand, for the model of a prolate spheroidal protrusion superimposed on a planar surface, we have considered the situation where the two axes of the spheroid become equal $(\alpha=1.0)$. This then is just a hemisphere superimposed on a planar surface. The relevant quantities were then calculated and the results matched extremely well.

In figure 2, for the model of a hemisphere superimposed on an hyperpoloid, the typical behaviour of the ion's lateral displacement with respect to the tip symmetry axis ( $x$ coordinate) at departure position (close to the sample) and arrival position (at screen or detector) is shown. At each position of the ion, along its trajectory, let us call the angular coordinate (with respect to the tip symmetry axis) of its velocity by $\theta$. Similarly, let us call $\sigma$ the corresponding angular coordinate for the local electric field along the ion's trajectory. In figure 3, for the same model as figure 2, the variation of these angular coordinates along a typical trajectory for the ion are shown. Similarly, for the model of a prolate spheroid superimposed on a planar surface, we present in figure 4 the $x$-coordinates of the ion, at the screen and at the departure positions, while in figure 5 the typical behaviour of the angular coordinates, $\theta$ and $\sigma$, for the ion's velocity and for the local electric field along its trajectory, are shown.

It is possible to observe that, irrespective to the adopted model, the lateral displacement at the screen is greater 


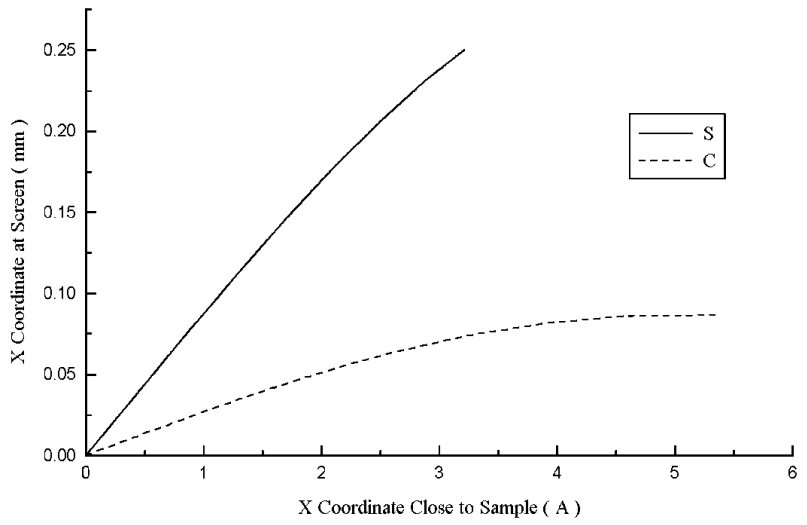

(a)

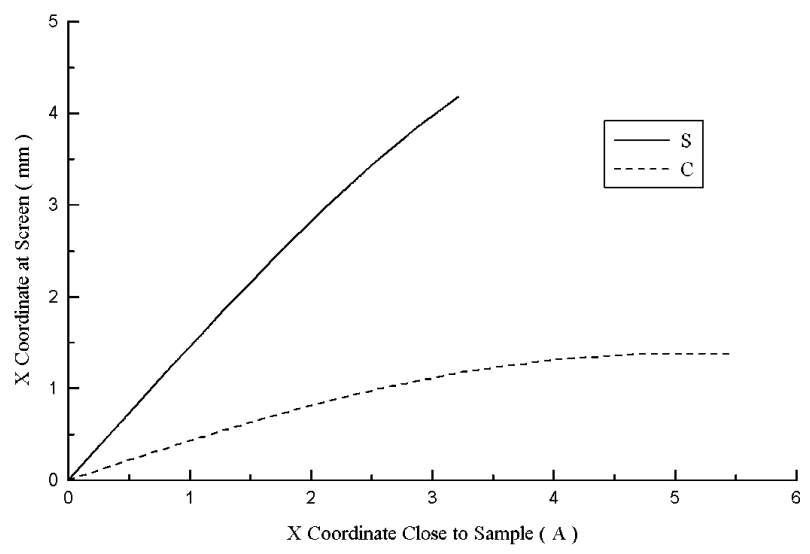

(b)

Figure 2. Hyperboloid plus a hemisphere model. Lateral displacement (the ion's $x$-coordinate) with respect to the tip axis, at its departure position and at its arrival position at the screen (or detector). This is assumed to be $12 \mathrm{~cm}$ away from the tip, which in turn has a hemisphere of radius $5.0 \AA$. (a) For $R_{t}=1000 \AA$; (b) for $R_{t}=200 \AA$. In both cases the departure position corresponds to an angular coordinate which varies from 0 to $40^{\circ}$. The departure position from a point at the critical surface and from a point at the electrical surface of the metal are indicated by $\mathrm{C}$ and $\mathrm{S}$, respectively.

for a particle which departs from the sample surface than for a one generated at the critical distance (see figures 2 and 4). This is consistent with the experimental observation accordingly to which 'the trajectories of ions evaporating from protruding particles ... diverge more than the trajectories of the gas ions which form the corresponding FIM image' (Miller et al 1996), with consequences on the screen image size of particles (Waugh et al 1976). It is possible to observe a trend in figure 2 , which is confirmed in figure 4 , on the occurrence of a maximum lateral displacement at the screen as a function of the angular coordinate at the departure position. Along the protrusion surface, and also along the 'bump' that it causes in the critical surface, the electric field is not constant, decreasing as the angular coordinate increases. So, there is a trade-off between two factors: increasing the departure angle would lead to an increase in the lateral displacement provided the electric field was the same, but as this is not the case and since the field decreases, this possibility of a maximum occurs.

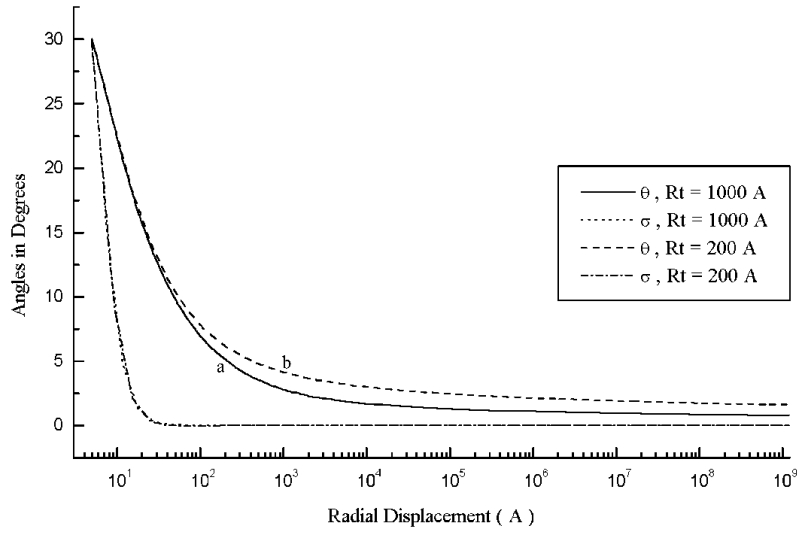

Figure 3. Hyperboloid plus hemisphere model. Angular coordinates, $\theta$ and $\sigma$ (see section 3 ) corresponding, respectively, to the ion's velocity and local electric field. The departure position, from rest and at the surface, corresponds to an angular coordinate equal to $30^{\circ}$. (a) For $R_{t}=1000 \AA$; (b) for $R_{t}=200 \AA$. In both cases the hemispherical protrusion has a radius equal to $5.0 \AA$. It is possible to observe that, despite the difference in the values of $R_{t}$ by a factor of five, the angle $\sigma$ for the electric field is essentially the same. A small difference is noticeable at points close to the sample, which cannot be distinguished on this scale.

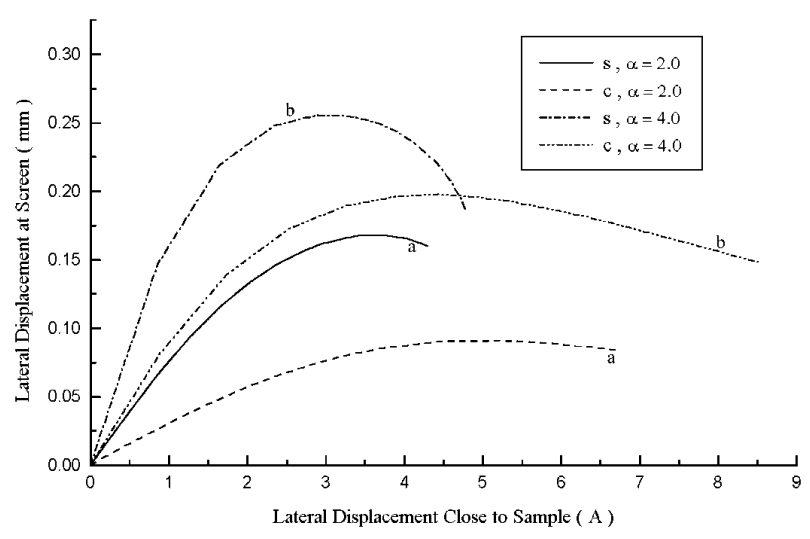

Figure 4. Planar surface plus a hemispherical prolate spheroid model. Lateral displacement (the ion's $x$-coordinate) with respect to the tip axis, at its departure position and at its arrival position at the screen (or detector). This is assumed to be $12 \mathrm{~cm}$ away from the sample, which in turn has a hemispherical prolate spheroid of smaller radius equal to $5.0 \AA$, superimposed on a planar surface. (a) For $\alpha=2.0$; (b) for $\alpha=4.0$. In both cases the departure position corresponds to an angular coordinate which varies from 0 to $40^{\circ}$. The departure position from a point at the critical surface and from a point at the electrical surface of the metal are indicated by $\mathrm{C}$ and $\mathrm{S}$, respectively.

In figures 3 and 5 it can be seen that, for both adopted models, the angle between the field direction and the $z$ axis (angle $\sigma$ ) is always smaller than the corresponding angle for the ion's velocity (angle $\theta$ ). This, of course, results from inertia, since the ion, when close to the protrusion, is accelerated away from the sample's symmetry axis retaining a lateral momentum while the direction of the electric field rapidly changes with the decreasing influence of the protrusions in its local direction and intensity. When the protrusion is on a hyperboloid, the long-range effect of the hyperboloid curvature (instead of a plane) can be seen, as in figure 3. This does not occur 


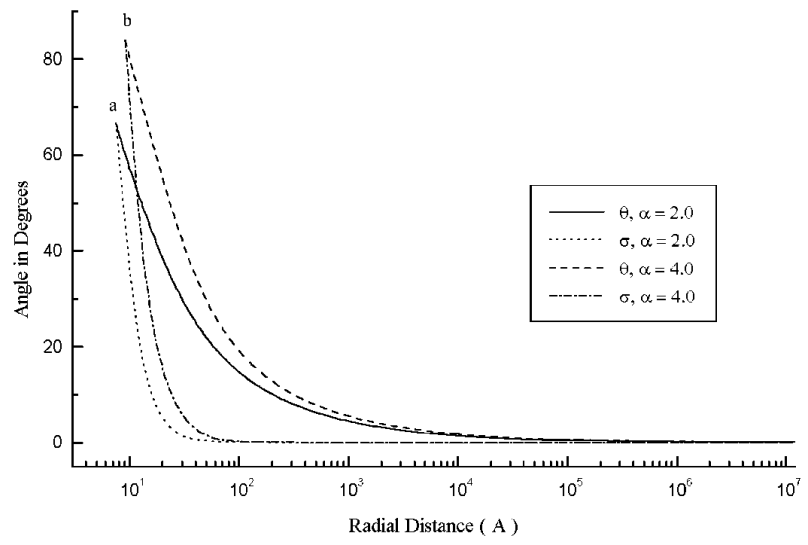

Figure 5. Planar surface plus a hemispherical prolate spheroid model. Angular coordinates, $\theta$ and $\sigma$ (see section 3) corresponding to the ion's velocity and local electric field, respectively. The departure position, from rest and at the surface, corresponds to an angular coordinate equal to $30^{\circ}$ : (a) for $\alpha=2.0$; (b) for $\alpha=4.0$. In both cases the prolate hemispherical protrusion has a smaller radius equal to $5.0 \AA$.

in figure 5, even for protrusions with a different ratio between their longitudinal and transversal dimensions. In this case, for greater distances from the sample, the two curves for $\theta$ coincide, with the same occurring for the $\sigma$ curves.

\section{Conclusions}

We have been able to use simplified models for calculating ion trajectories as they occur in the AP-FIM and in GFIS. The experimental results of observations of the images produced by field ionization are compared with those produced by evaporation/desorption for the same area of a sample. Typical figures obtained for the differences in positions i.e. from a few tenths of a millimetre to a few millimetres, at screen (or detector) for an ion produced by field ionization and another produced by field evaporation (or desorption), are in good agreement with experimental results. To improve the models, specific and more systematic measurements are, as far as it was possible to find in the literature, still lacking.

The present interpretation for the reduced beam ion divergence obtained when a small protrusion is grown upon a standard field emitter tip is claimed to be the result of a self-focusing process of the ion's trajectories. It seems to be possible to make, from the present results, an additional comment. Our calculations in fact show this self-focusing process, which is a direct result of the local electric field direction and intensity. However, this is also favoured from the fact that in the GFIS the ions (supplied from the shank towards the protruding area) result from field ionization. Hence, most of the ionization takes place in an area that is at a fraction of nanometre above the protruding surface, where, besides being more easily ionized as a result of a higher electric field (compared with a region just above a smoother part of the sample) they also have a subsequent trajectory which results in a smaller lateral displacement at the screen/detector, as our results clearly show.

\section{Acknowledgments}

I would like to express my gratitude to the Royal Society and to Dr R M Lambert and his group for, respectively, support and hospitality in Cambridge, where this work could be finished. The author acknowledges the benefit of discussions and comments by Dr M K Miller and Dr R G Forbes. The help of Mr J J Cowell is also acknowledged. This work has financial support of CNPq and CAPES (Brazilian agencies).

\section{References}

Bono S and Good R H 1983 Surf. Sci. 134272

Borisov V T, Kirienko V I and Potapov L P 1968 Instrum. Exp. Tech. 4952

Brenner S S and McKinney J J 1970 Surf. Sci. 2388

de Castilho C M C and Kingham D R 1986 Surf. Sci. 17375

Gillot L and Sugden J 1973 J. Phys. E: Sci. Instrum. 61218

Homeier H H H and Kingham D R 1983 J. Phys. D: Appl. Phys. $16 \mathrm{~L} 115$

Jousten K, Böhringer K and Kalbitzer S 1988 Appl. Phys. B 46313

Kalbitzer S and Knoblauch A 1998 Rev. Sci. Instrum. 691026

Miller M K, Cerezo A, Hetherington M G and Smith G D W 1996 Atom Probe Field Ion Microscopy (Oxford: Clarendon)

Morse P M and Feshbach H 1953 Methods of Theoretical Physics (New York: McGraw-Hill)

Panitz J A 1978 Prog. Surf. Sci. 8219

Rose D J 1956 J. Appl. Phys. 27215

Schelkunoff S A 1961 Applied Mathematics for Engineers and Scientists (Princeton: Van Nostrand)

Smith R and Walls J M 1978 J. Phys. D: Appl. Phys. 11409

Waugh A R, Boyes E D and Southon M J 1976 Surf. Sci. 61109 